\title{
Rigid components in molecular graphs
}

\author{
Bill Jackson*and Tibor Jordán ${ }^{\dagger}$ \\ Revised version, November 2, 2006
}

\begin{abstract}
In this paper we consider 3-dimensional generic bar-and-joint realizations of squares of graphs. These graphs are also called molecular graphs due to their importance in the study of flexibility in molecules. The Molecular Conjecture, posed in 1984 by T-S. Tay and W. Whiteley, indicates that determining rigidity (or more generally, computing the number of degrees of freedom) of molecular graphs may be tractable by combinatorial methods. We show that the truth of the Molecular Conjecture would imply an efficient algorithm to identify the maximal rigid subgraphs of a molecular graph. In addition, we prove that the truth of two other conjectures in combinatorial rigidity (due to A. Dress and D. Jacobs, respectively) would imply the truth of the Molecular Conjecture.
\end{abstract}

\section{Introduction}

Many important aspects of the flexibility of molecules can be studied using the theory of combinatorial rigidity. This branch of mathematics, which has strong links to graph theory and geometry, is concerned with the rigidity properties (i.e. number of degrees of freedom, maximal rigid components, redundant elements, etc.) of different geometric structures, such as bar-and-joint, body-and-hinge, and body-and-bar frameworks. For 'generic' frameworks these properties depend only on the graph associated to the

\footnotetext{
${ }^{*}$ School of Mathematical Sciences, Queen Mary, University of London, Mile End Road, London E1 4NS, England. e-mail: b.jackson@qmul.ac.uk

${ }^{\dagger}$ Department of Operations Research, Eötvös University, Pázmány Péter sétány 1/C, 1117 Budapest, Hungary. e-mail: jordan@cs.elte.hu. Supported by the MTA-ELTE Egerváry Research Group on Combinatorial Optimization and the Hungarian Scientific Research Fund grant no. T049671.
} 

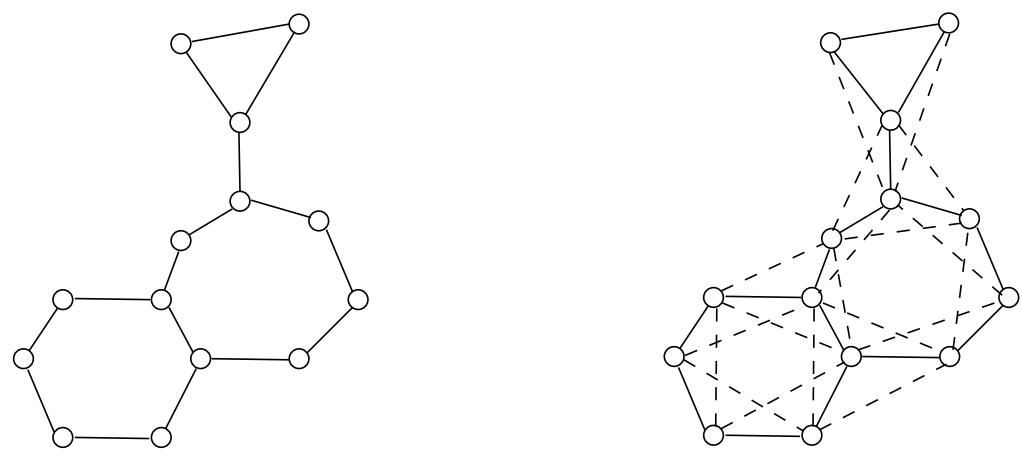

Figure 1: A graph $G$ and its square $G^{2}$.

framework, in which vertices correspond to joints, or bodies, and edges correspond to the bars, or hinges, of the framework which connect them.

Given a molecular structure made up of atoms and bonds in 3-space, we can extract a geometric structure for the molecule whose flexibility can be analyzed by combinatorial methods on the associated graph [24]. These mathematical models and the related combinatorial algorithms are the basis for the 'pebble game algorithms' used in FIRST, ROCK, and other fast algorithms for predicting the flexibility and rigidity of discrete molecular structures arising in proteins and glasses $[11,12,13,18]$. In this paper we focus on the problem of determining the rigidity properties of the bar-andjoint model of molecules and the corresponding combinatorial problems on graphs.

We model a molecule made up of atoms and covalent bonds as a graph $G$ in which vertices represent atoms and edges represent bonds. Since the angles between the covalent bonds of each atom are fixed (as well as the distance between bonded atoms) we need to add additional 'bond bending' edges connecting each pair of vertices of distance two in $G$, to fix these angles. This forms the square of the graph $G$, which we denote by $G^{2}$, see Figure 1. Squares of graphs are sometimes called molecular graphs or bond bending networks.

The Molecular Conjecture, due to Tay and Whiteley [17, Conjecture 1], asserts that the square $G^{2}$ of a graph $G$ is rigid in 3-space if and only if $5 G$, the graph obtained from $G$ by replacing each edge $e$ by five copies of $e$, contains six edge-disjoint spanning trees. This conjecture indicates that 
the problem of determining when a molecular graph is rigid (or more generally, computing its number of degrees of freedom, or floppy modes, and its maximal rigid subgraphs) may be tractable by combinatorial methods. For a substantial collection of supporting evidence for the Molecular Conjecture see [24].

Our main result is a new combinatorial algorithm for computing the number of degrees of freedom and determining the maximal rigid subgraphs in a molecular graph $G^{2}$, which runs on the underlying graph $G$. We give a proof of correctness of our algorithm based on new structural properties of molecular graphs and of graphs containing six edge-disjoint spanning trees, as well as on the assumption that the Molecular Conjecture is true.

Our motivation is to establish that the truth of the Molecular Conjecture would imply that there is a fast, provably correct algorithm for computing the number of degrees of freedom and determining the maximal rigid subgraphs in a molecular graph. The algorithm of Jacobs [11, 12], which was used in the first versions of the FIRST software, uses a different approach for computing the degrees of freedom and the maximal rigid subgraphs. It runs on $G^{2}$, rather than $G$, and is a modification of the 'pebble game' algorithm, originally designed for rigidity analysis of 2-dimensional frameworks. There is no rigorous proof for its correctness even if we assume that the Molecular Conjecture is true. A different algorithm for rigidity of molecular graphs is given by Franzblau [3]. Her algorithm only gives upper and lower bounds on the number of degrees of freedom of a molecular graph (but she shows that her bounds are valid without assuming that the Molecular Conjecture is true).

To provide more evidence in favour of the Molecular Conjecture, we also use our structural results to show that the truth of two other conjectures in combinatorial rigidity (due to Dress and Jacobs, respectively) would imply the truth of the Molecular Conjecture.

\section{Rigid graphs and the Molecular Conjecture}

All graphs considered are finite and without loops. We reserve the term graph for graphs without multiple edges and refer to graphs which may contain multiple edges as multigraphs. A framework $(G, p)$ (in 3-space) is a graph $G=(V, E)$ and a map $p: V \rightarrow \mathbb{R}^{3}$. We will also say that $(G, p)$ is a realization of $G$. The rigidity matrix of the framework is a matrix $R(G, p)$ of size $|E| \times 3|V|$. For each edge $v_{i} v_{j} \in E$, the entries in the 
row corresponding to the edge $v_{i} v_{j}$ are defined as follows: the 3 columns corresponding to the vertex $v_{i}$ contain the 3 coordinates of $p\left(v_{i}\right)-p\left(v_{j}\right)$; the 3 columns corresponding to the vertex $v_{j}$ contain the 3 coordinates of $p\left(v_{j}\right)-p\left(v_{i}\right)$; the remaining entries are all zeros. See [20] for more details.

We refer to the vectors in the null space of $R(G, p)$ as infinitesimal motions of $(G, p)$. This definition is motivated by the fact that each continuous motion of $(G, p)$ which preserves all 'edge lengths' gives rise to an infinitesimal motion [20]. The null space of $R(G, p)$ will have dimension at least six since the translations and rotations of $\mathbb{R}^{3}$ give rise to six linearly independent infinitesimal motions (when $|V| \geq 3$ ). Thus, when $|V| \geq 3$, the rank of $R(G, p)$ is at most $3|V|-6$. We say $(G, p)$ is infinitesimally rigid if the dimension of the null space of $R(G, p)$ is equal to six (or equivalently, if the rank of $R(G, p)$ is equal to $3|V|-6)$.

A framework $(G, p)$ is generic if the ranks of its rigidity matrix, and all its submatrices, are maximum among all realizations of $G$. It is known that almost all realizations of $G$ are generic, and that each infinitesimal motion of a generic framework is 'induced' by a continuous motion of the framework. We denote the rank of the rigidity matrix of a generic realization of $G$ by $r(G)$. By the preceding paragraph $r(G) \leq 3|V|-6$, whenever $|V| \geq 3$. The graph $G$ is said to be rigid if either $G$ is a complete graph on at most two vertices, or $|V| \geq 3$ and $r(G)=3|V|-6$. More generally $3|V|-r(G)$, i.e. the dimension of the null space of $R(G, p)$ for a generic realization $(G, p)$ of $G$, is referred to as the number of degrees of freedom of $G$, and measures the flexibility of the graph.

It is a difficult open problem to determine which graphs are rigid. For a survey and partial results see $[3,6,7,8,9,20]$. The Molecular Conjecture, due to Tay and Whiteley [17, Conjecture 1], see also [20, 21, 22, 23, 24], indicates that the problem of determining when molecular graphs are rigid may be significantly easier than the problem for arbitrary graphs. Conjectures 2.1 and 2.2 below are the bar-and-joint versions of the Molecular Conjecture. Recall that the square of a graph $G=(V, E)$ is denoted by $G^{2}$, and the multigraph obtained from $G$ by replacing each edge $e \in E$ by five copies of $e$ is denoted by $5 G$.

Conjecture 2.1 Let $G$ be a graph with minimum degree at least two. ${ }^{1}$ Then $G^{2}$ is rigid if and only if $5 G$ contains six edge-disjoint spanning trees.

\footnotetext{
${ }^{1}$ The conjecture becomes false if we allow vertices of degree one. This is not a significant constraint, however, since it would be straightforward to extend the characterization to allow such vertices if the conjecture was known to be true, see [10].
} 
The 'defect form' of Conjecture 2.1 is the following. Let $G=(V, E)$ be a graph. For a family $\mathcal{F}$ of pairwise disjoint subsets of $V$ let $E_{G}(\mathcal{F})$ denote the set, and $e_{G}(\mathcal{F})$ the number, of edges of $G$ connecting distinct members of $\mathcal{F}$. For a partition $\mathcal{P}$ of $V$ let

$$
\operatorname{def}_{G}(\mathcal{P})=6(|\mathcal{P}|-1)-5 e_{G}(\mathcal{P})
$$

denote the deficiency of $\mathcal{P}$ in $G$ and let

$$
\operatorname{def}(G)=\max \left\{\operatorname{def}_{G}(\mathcal{P}): \mathcal{P} \text { is a partition of } V\right\} .
$$

Note that $\operatorname{def}(G) \geq 0$ since $\operatorname{def}_{G}(\{V\})=0$. Nash-Williams [14] and Tutte [19] independently characterized when a graph has $k$ edge-disjoint spanning trees. Their result implies that $5 G$ has six edge-disjoint spanning trees if and only if $\operatorname{def}(G)=0$.

Conjecture 2.2 [10] Let $G=(V, E)$ be a graph with minimum degree at least two. Then

$$
r\left(G^{2}\right)=3|V|-6-\operatorname{def}(G) .
$$

Since there are efficient algorithms for computing $\operatorname{def}(G)$, Conjecture 2.2 would imply that $r\left(G^{2}\right)$, and hence the number of degrees of freedom of $G^{2}$, can be computed efficiently. We will return to this in Section 6 .

We showed in [10] that Conjectures 2.1 and 2.2 are equivalent. We also showed that the right hand side of (1) is an upper bound on $r\left(G^{2}\right)$.

Theorem 2.3 [10] Let $G=(V, E)$ be a graph of minimum degree at least two. Then

$$
r\left(G^{2}\right) \leq 3|V|-6-\operatorname{def}(G) .
$$

\section{$3 \quad$ Basic results on graph rigidity}

Let $H=(V, E)$ be a multigraph. For $X \subseteq V$, let $E_{H}(X)$ denote the set, and $i_{H}(X)$ the number, of edges in $H[X]$, that is, in the subgraph induced by $X$ in $H$. For $X \subset V$ let $d_{H}(X)=e_{H}(X, V-X)$ denote the degree of $X$. If $X=\{v\}$ for some $v \in V$ then we simply write $d_{H}(v)$ for the degree of $v$. The set of neighbours of $X$ (i.e. the set of those vertices $v \in V-X$ for which there exists an edge $u v \in E$ with $u \in X)$ is denoted by $N_{H}(X)$. We use $E(X), i(X), d(X)$, or $N(X)$ when the multigraph $H$ is clear from the context. 

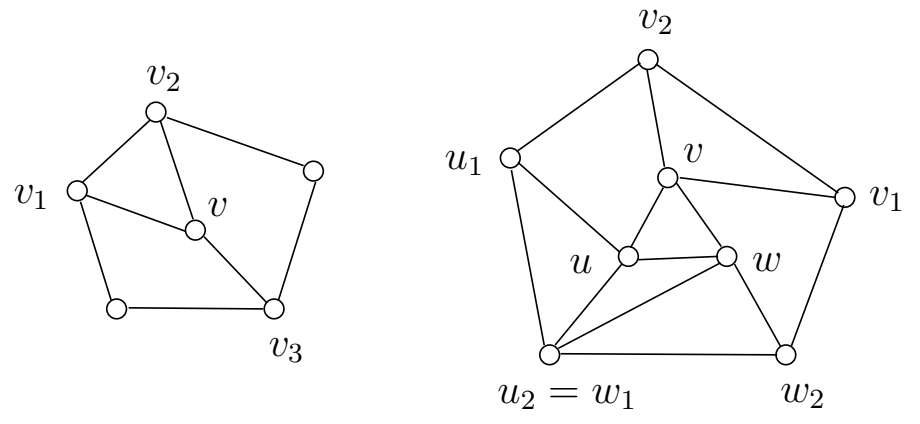

Figure 2: The graphs obtained by applying the operations of Lemma 3.1 and Lemma 3.2 to a cycle of length five.

We say that a set of edges $E^{\prime} \subseteq E$ is $M$-independent if the rows of the rigidity matrix $R(H, p)$ corresponding to $E^{\prime}$, are linearly independent in a generic realization $(H, p)$ of $H$. The set of edges $E^{\prime}$ is an $M$-basis for $H$ if $E^{\prime}$ is a maximum set of $M$-independent edges of $H$. (Thus every $M$-independent set of edges can be extended to an $M$-basis, every $M$-basis contains $r(H)$ edges and $H$ is rigid if and only if $H$ has an $M$-independent set of $3|V|-6$ edges, when $|V| \geq 3$.) We say that the graph $H$ is $M$-independent if its edge-set $E$ is $M$-independent.

We shall use the following concepts and basic results from rigidity theory.

Lemma 3.1 [20, Lemma 9.1.3] Let $H=(V, E)$ be a graph and $v_{1}, v_{2}, \ldots v_{s}$ be distinct vertices of $H$ for some $s \in\{1,2,3\}$. Let $G$ be obtained from $H$ by adding a new vertex $v$ and all edges $v v_{i}$ for $1 \leq i \leq s$. Then $G$ is $M$-independent if and only if $H$ is $M$-independent.

Lemma 3.2 [10] Let $H=(V, E)$ be an $M$-independent graph and $\left\{u_{1}, u_{2}\right\}$, $\left\{v_{1}, v_{2}\right\}$ and $\left\{w_{1}, w_{2}\right\}$ be three sets of distinct vertices of $H$ with $\left|\left\{u_{1}, u_{2}, v_{1}, v_{2}, w_{1}, w_{2}\right\}\right| \geq 3$. Let $G$ be obtained from $H$ by adding three new vertices $u, v, w$, the edges $u v, v w, u w$, and all edges $u u_{i}, v v_{i}, w w_{i}$ for $1 \leq i \leq 2$. Then $G$ is $M$-independent.

The operations of Lemmas 3.1 and 3.2 are illustrated in Figure 2. The next result follows from the 'Glueing Lemma' [20, Lemma 11.1.9]. 
Lemma 3.3 Let $H_{1}, H_{2}$ be rigid graphs with at least three vertices and $H=$ $H_{1} \cup H_{2}$. Then

(a) $H$ is rigid if and only if $\left|V\left(H_{1}\right) \cap V\left(H_{2}\right)\right| \geq 3$.

(b) If $\left|V\left(H_{1}\right) \cap V\left(H_{2}\right)\right|=2, u \in V\left(H_{1}\right)-V\left(H_{2}\right)$, and $v \in V\left(H_{2}\right)-V\left(H_{1}\right)$, then $H+u v$ is rigid.

A rigid component of a graph $H=(V, E)$ is a rigid subgraph of $H$ which is maximal with respect to inclusion. Each rigid component is clearly an induced subgraph of $H$. For $u, v \in V$, the edge $u v$ is an implied edge of $H$ if $u v \notin E$ and $r(H+u v)=r(H)$. The closure $\hat{H}$ of $H$ is the graph obtained by adding all the implied edges to $H$. A rigid cluster of $H$ is a set of vertices which induce a maximal complete subgraph of $\hat{H}$. (Informally we may think of a rigid cluster of $H$ as a maximal subset $U$ of $V$ with the property that every continuous motion of a generic realization $(H, p)$ of $H$ which preserves the lengths of all edges $v w \in E$, also preserves the distances between the points $p(u), p\left(u^{\prime}\right)$ for all $u, u^{\prime} \in U$.)

It is clear that (the vertex set of) each rigid component of $H$ is contained in a rigid cluster of $H$. In general $H$ can have rigid clusters which are not even vertex sets of rigid subgraphs of $G$, see Figure $3 .^{2}$ Jacobs [11] has conjectured, however, that the rigid clusters of a molecular graph are the same as the vertex sets of its rigid components ${ }^{3}$.

Conjecture 3.4 Let $G=(V, E)$ be a graph and $U \subseteq V$. Then $U$ is a rigid cluster of $G^{2}$ if and only if $G^{2}[U]$ is a rigid component of $G^{2}$.

In order to verify Conjecture 3.4, we need to show that $r\left(G^{2}+u v\right)=$ $r\left(G^{2}\right)$ if and only if $u, v$ belong to the same rigid component of $G^{2}$. It seems that it would be difficult to verify this even if we assume that the Molecular Conjecture is true, since the graph $G^{2}+u v$ need not be a molecular graph.

A cover of a graph $H=(V, E)$ is a collection $\mathcal{X}$ of subsets of $V$, each of size at least two, such that $\cup_{X \in \mathcal{X}} E(X)=E$. A cover $\mathcal{X}=\left\{X_{1}, X_{2}, \ldots, X_{m}\right\}$ of $H$ is 2-thin if $\left|X_{i} \cap X_{j}\right| \leq 2$ for all $1 \leq i<j \leq m$. For $X_{i} \in \mathcal{X}$ let $f\left(X_{i}\right)=1$ if $\left|X_{i}\right|=2$ and $f\left(X_{i}\right)=3\left|X_{i}\right|-6$ if $\left|X_{i}\right| \geq 3$. Given a 2-thin cover $\mathcal{X}$ of $H$, let $\Theta(\mathcal{X})$ be the set of all pairs of vertices $u v$ such that $X_{i} \cap X_{j}=\{u, v\}$ for

\footnotetext{
${ }^{2}$ Note that the graph on Figure 3 is not a square. This follows, for example, from the simple observation that if $H=G^{2}$ for some graph $G$ and $H-\{u, v\}$ is disconnected for some pair $u, v \in V(G)$, then we would have $u v \in E(H)$.

${ }^{3}$ This conjecture is actually a combination of two assertions [11, Observation 3.1] and [11, Theorem 4.3] whose proofs are incomplete.
} 


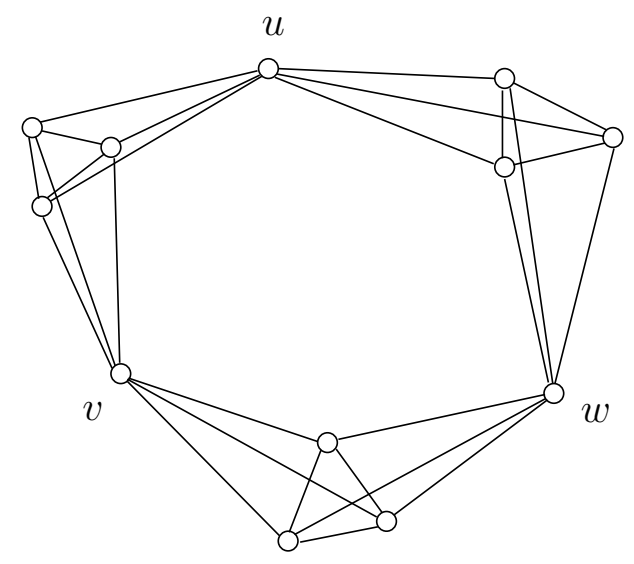

Figure 3: A graph $H$ with a rigid cluster $U=\{u, v, w\}$ for which $H[U]$ is not rigid.

some $1 \leq i<j \leq m$. For each $u v \in \Theta(\mathcal{X})$ let $\theta(u v)$ be the number of sets $X_{i}$ in $\mathcal{X}$ such that $\{u, v\} \subseteq X_{i}$ and put

$$
\operatorname{val}(\mathcal{X})=\sum_{X \in \mathcal{X}} f(X)-\sum_{u v \in \Theta(\mathcal{X})}(\theta(u v)-1) .
$$

Using Lemma 3.3(a), we can see that any two rigid clusters of $G$ intersect in at most two vertices. Thus the set of rigid clusters of $G$ is a 2-thin cover of $G$. Dress conjectured in 1987 that the value of this special 2-thin cover is equal to the rank of $G$.

Conjecture 3.5 (see [6, Conjecture 5.6.1],[2], and [16, Conjecture 2.3]) Let $H=(V, E)$ be a graph and $\mathcal{X}$ be the set of rigid clusters of $H$. Then

$$
r(H)=\operatorname{val}(\mathcal{X}) .
$$

We will show in Section 5 that the Molecular Conjecture would follow from the truth of Conjectures 3.4 and 3.5.

\section{Rigid components of molecular graphs}

Let $G$ be a graph. We first verify a number of properties of the rigid components of $G^{2}$. We then use these to show that the vertex sets of the rigid 
components of $G^{2}$ are a 2-thin cover of $G^{2}$ whose value gives an upper bound on $r\left(G^{2}\right)$. We conjecture that this upper bound is tight. Throughout this section we refer to the edges in $E\left(G^{2}\right)-E(G)$ as new edges of $G^{2}$.

Lemma 4.1 Suppose $G$ is a connected graph with at least three vertices. Let $C$ be a rigid component of $G^{2}$ and let $Y=V(C)$. Then

(a) $|Y| \geq 3$,

(b) $d_{G[Y]}(v) \geq 1$ for all $v \in Y$,

(c) $G[Y]$ is connected,

(d) $d_{G[Y]}(v)=1$ for all $v \in N_{G}(V-Y)$.

Proof: (a) This follows from the fact that each edge of $G^{2}$ belongs to a triangle and hence to a rigid subgraph with three vertices.

(b) Suppose that all the edges incident to $v$ in $G^{2}[Y]$ are new edges. The new edges of $G^{2}$ are 'generated' by pairs of edges of $G$, and, by our assumption, no edge from any of these pairs can be in $G[Y]$.

First suppose $|Y| \geq 4$. Since $G^{2}[Y]$ is rigid, there must be (at least) three new edges $e, f, g$ incident to $v$ in $G^{2}[Y]$. By considering the pairs of edges of $G$ which 'generate' $e, f, g$, and the edges that these pairs 'generate' in $V-Y$, it is easy to check that either there is a vertex $y \in V-Y$ connected to $Y$ by three edges in $G^{2}$, or there is a triangle $T$ in $G^{2}-Y$ which satisfies the hypotheses of Lemma 3.2 in $G^{2}$. This contradicts the maximality of $C$ by Lemma 3.1 or Lemma 3.2.

The proof for the case when $|Y|=3$ proceeds similarly by considering the two new edges $e, f$ incident to $v$ in $G^{2}$ as well as the third edge $g$ of $G^{2}[Y]$.

(c) Consider a connected component $D$ of $G[Y]$. By (b) each vertex of $D$ is incident to an edge in $G[Y]$. Let $u v, v w$ be a pair of edges in $E$ which 'generate' a new edge $u w$ of $G^{2}$ with $u \in V(D)$ and $w \in Y-V(D)$. We must have $v \in V-Y$. Since $d_{G[Y]}(w) \geq 1$ by (b), $v$ must be connected to $Y$ by at least three edges in $G^{2}$. This contradicts the maximality of $C$ by Lemma 3.1.

(d) Let $u v \in E$ with $v \in Y$ and $u \in V-Y$. We have $d_{G[Y]}(v) \geq 1$ by (b). If $d_{G[Y]}(v) \geq 2$ then $u$ must be connected to $Y$ by at least three edges in $G^{2}$, a contradiction by Lemma 3.1 and the maximality of $C$. Thus $d_{G[Y]}(v)=1$. 
Lemma 4.2 Suppose $G$ is a connected graph with at least three vertices and that $G^{2}$ is not rigid. Let $C_{1}, C_{2}$ be distinct rigid components of $G^{2}$ with $Y_{1}=V\left(C_{1}\right), Y_{2}=V\left(C_{2}\right)$ and $Y_{1} \cap Y_{2}=\{u, v\}$. Then

(a) $u v \in E$,

(b) $d_{G\left[Y_{1}\right]}(u)=1$ and $d_{G\left[Y_{2}\right]}(v)=1 \quad\left(\right.$ or $d_{G\left[Y_{2}\right]}(u)=1$ and $\left.d_{G\left[Y_{1}\right]}(v)=1\right)$,

(c) uv is contained in no rigid components of $G$ other than $C_{1}, C_{2}$.

Proof: The maximality of $C_{1}, C_{2}$ implies that $Y_{1}-Y_{2} \neq \emptyset \neq Y_{2}-Y_{1}$. Part (a) follows from Lemma 4.1(b), Lemma 3.3(b), and the maximality of $C$. Part (b) follows in a similar way from Lemma 3.3(b). Part (c) follows from (b) and Lemma 4.1(a),(c).

Lemma 4.3 Suppose $G$ is a connected graph with at least three vertices and that $G^{2}$ is not rigid. Let $C_{1}, C_{2}$ be distinct rigid components of $G^{2}$ with $Y_{1}=V\left(C_{1}\right), Y_{2}=V\left(C_{2}\right)$ and $Y_{1} \cap Y_{2}=\{v\}$. Then $d_{G\left[Y_{1}\right]}(v)=1=d_{G\left[Y_{2}\right]}(v)$.

Proof: The lemma follows from Lemma 4.1(b), Lemma 3.1, and the maximality of $C$.

Let $H$ be a graph without isolated vertices. It follows from the fact that every edge of $H$ is contained in a rigid component, and Lemma 3.3(a), that the vertex sets of the rigid components $H$ form a 2-thin cover of $H$. We will abuse our notation and use $\Theta(\mathcal{C})$ and $\operatorname{val}(\mathcal{C})$ to denote, respectively, the hinge set and the value of this 2 -thin cover.

Theorem 4.4 Suppose $G$ is a connected graph with at least three vertices and that $G^{2}$ is not rigid. Let $\mathcal{C}=\left\{C_{1}, C_{2}, \ldots, C_{t}\right\}$ be the set of rigid components of $G^{2}$. For all $1 \leq i \leq t$, let $Y_{i}=V\left(C_{i}\right)$ and let $Q_{i}=Y_{i}-\{v$ : $d_{G\left[Y_{i}\right]}(v)=1$ and $\left.d_{G}(v) \geq 2\right\}$. Put $\mathcal{Q}=\left\{Q_{1}, Q_{2}, \ldots, Q_{t}\right\}$. Then

(a) $\mathcal{Q}$ is a partition of $V$,

(b) $\operatorname{val}(\mathcal{C})=3|V|-6-\operatorname{def}_{G}(\mathcal{Q})$, and

(c) $r\left(G^{2}\right) \leq \operatorname{val}(\mathcal{C})$.

Proof: (a) By Lemma 4.1(a),(c), $Q_{i} \neq \emptyset$ for all $1 \leq i \leq t$. Choose $v \in V$. We first suppose that $d_{G}(v)=1$. It is easy to see that $v$ is contained in a unique rigid component $C_{i} \in \mathcal{C}$ and that $X_{i}$ is the unique set in $\mathcal{Q}$ which contains $v$. Next we suppose that $d_{G}(v) \geq 2$. Then there is a unique rigid component $C_{i} \in \mathcal{C}$ which contains $\{v\} \cup N_{G}(v)$. We have $v \in X_{i}$ and, by Lemmas 4.2 (b) and 4.3, no other set in $\mathcal{Q}$ can contain $v$. Thus $\mathcal{Q}$ partitions 
$V$.

(b) By Lemma 4.1(a) we have $\left|Y_{i}\right| \geq 3$ for all $1 \leq i \leq t$. By Lemma 4.2(a),(c) we have $u v \in E$ and $\theta(u v)=2$ for all $u v \in \Theta(\mathcal{C})$. By Lemma 4.2(b) and the definition of $\mathcal{Q}$ we have $E_{G}(\mathcal{Q})=\Theta(\mathcal{C})$. Lemma 3.1 implies that $N_{G}\left(Q_{i}\right) \subseteq Y_{i}$ and hence $\left|Y_{i}\right|=\left|Q_{i}\right|+d_{G}\left(Q_{i}\right)$ for all $1 \leq i \leq t$. Thus $\operatorname{val}(\mathcal{C})=$ $\sum_{C_{i} \in \mathcal{C}}\left(3\left|Y_{i}\right|-6\right)-|\Theta(\mathcal{C})|=3|V|+6 e_{G}(\mathcal{Q})-6 t-e_{G}(\mathcal{Q})=3|V|-6-\operatorname{def}_{G}(\mathcal{Q})$.

(c) Theorem 2.3 and (b) now imply

$$
r\left(G^{2}\right) \leq 3|V|-6-\operatorname{def}(G) \leq 3|V|-6-\operatorname{def}_{G}(\mathcal{Q})=\operatorname{val}(\mathcal{C}) .
$$

We will refer to the partition $\mathcal{Q}$ defined in Lemma 4.4 as the partition of $V$ generated by the rigid components of $G^{2}$.

It is easy to see that Theorem 4.4(c) implies that we have $r\left(G^{2}\right) \leq \operatorname{val}(\mathcal{C})$ for all graphs $G$ without isolated vertices. We conjecture that equality holds.

Conjecture 4.5 Let $G$ be a graph without isolated vertices. Then $r\left(G^{2}\right)=$ $\operatorname{val}(\mathcal{C})$.

We will show in the next section that this conjecture is equivalent to Conjecture 2.2. To do this we need to reduce the conjecture to the case of graphs of minimum degree at least two.

Lemma 4.6 If Conjecture 4.5 holds for all graphs of minimum degree at least two, then it holds for all graphs.

Proof: It is straightforward to show that if Conjecture 4.5 were false, then a smallest counterexample would have minimum degree at least two.

Note that there exist examples of a graph $H$ whose set of rigid components $\mathcal{C}$ satisfies $r(H)<\operatorname{val}(\mathcal{C}) .{ }^{4}$ It is conceivable, however, that the inequality $r(H) \leq \operatorname{val}(\mathcal{C})$ holds for all graphs $H$, not just molecular graphs.

\footnotetext{
${ }^{4}$ Let $G_{0}=\left(V_{0}, E_{0}\right)$ be a complete graph on five vertices with $V_{0}=\left\{v_{i}: 1 \leq i \leq 5\right\}$. For $1 \leq i<j \leq 5$ let $G_{i, j}=\left(V_{i, j}, E_{i, j}\right)$ be a complete graph on five vertices with $V_{i, j} \cap V_{0}=$ $\left\{v_{i}, v_{j}\right\}$ and $E_{i, j} \cap E_{0}=\left\{v_{i} v_{j}\right\}$ for $1 \leq i<j \leq 5$. Let $G=\left(G_{0} \cup\left(\bigcup_{1 \leq i<j \leq 5} G_{i, j}\right)\right)-E_{0}$. It can be seen that $r(G) \leq|E(G)|-1=89$. On the other hand, the set of rigid components of $G$ is $\mathcal{C}=\left\{G_{i, j}-v_{i} v_{j}: 1 \leq i<j \leq 5\right\}$ and we have $\operatorname{val}(\mathcal{C})=90$. See [9, Example 3] for more details.
} 

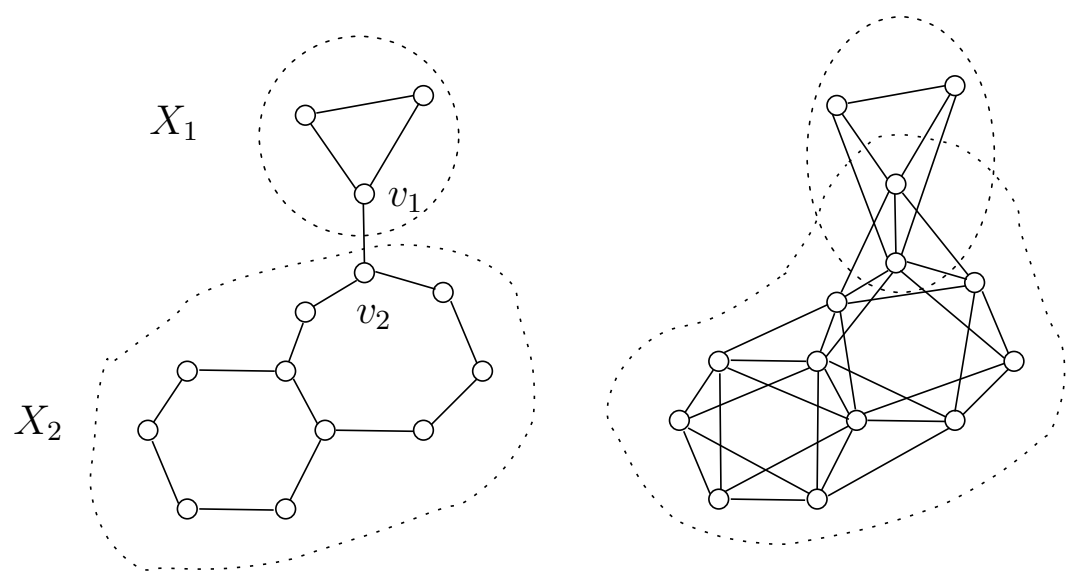

Figure 4: A graph $G$ and its brick partition $\left\{X_{1}, X_{2}\right\}$ together with its square $G^{2}$ and the vertex sets $\left\{X_{1}+v_{2}, X_{2}+v_{1}\right\}$ of the rigid components of $G^{2}$.

\section{$5 \quad$ Bricks and rigid components}

Let $G=(V, E)$ be a graph of minimum degree at least two. In this section, we consider the relationship between the partition $\mathcal{Q}$ of $V$ generated by the rigid components of $G^{2}$ and another partition of $V$. We say that the graph $G$ is strong if $5 G$ has six edge-disjoint spanning trees, or equivalently, if $\operatorname{def}(G)=0$. A subgraph $H$ is a brick of $G$ if $H$ is a maximal strong subgraph of $G$. Thus bricks are induced subgraphs of $G$. It was shown in [10] that the vertex sets of the bricks of $G$ partition $V$. We shall refer to this partition of $V$ as the brick partition of $G$. We illustrate the brick partition of a graph in Figure 4. We showed in [10] that the brick partition $\mathcal{B}$ of $G$ satisfies $\operatorname{def}_{G}(\mathcal{B})=\operatorname{def}(G)$. The first three lemmas of this section investigate the relationship between the bricks of $G$ and the rigid components of $G^{2}$. They will imply that $\mathcal{Q}$ is a refinement of $\mathcal{B}$, and that, if Conjecture 2.1 is true, then $\mathcal{Q}=\mathcal{B}$.

Henceforth, we shall use $V_{1}(H)$ to denote the vertices of degree one in a graph $H$.

Lemma 5.1 Let $G$ be a graph of minimum degree at least two, $C$ be a rigid component of $G^{2}, Y=V(C)$ and $X=Y-V_{1}(G[Y])$. Then $G[X]^{2}$ is rigid 
and $G[X]$ is strong.

Proof: Since $C$ is a rigid component of $G^{2}$, it follows from Lemma 3.1, Lemma 4.1 (a),(c), and the maximality of $C$ that $|Y| \geq 3, G[Y]$ is connected, and $G^{2}[Y]=G[Y]^{2}$. Since $Y-X=V_{1}(G[Y])$, we also have $G[X]$ is connected and $G^{2}[X]=G[X]^{2}$. Furthermore, either $|X|=1$, or $|X| \geq 3$ and $d_{G[X]}(v) \geq 2$ for all $v \in X$ (since the end-vertices of a cut-edge in $G[X]$ would form a separating pair in $C$, and, by Lemma 3.3(a), this would contradict the fact that $C$ is rigid). If $|X|=1$ then $G[X]^{2}$ is rigid and $G[X]$ is strong by definition. Hence we may suppose that $|X| \geq 3$.

Consider a vertex $v \in X$ with a neighbour in $Y-X$ and let $W$ be the set of all neighbours of $v$ in $Y-X$. Then $d_{G[X]}(v) \geq 2$, so $G^{2}\left[\{v\} \cup N_{G[X]}(v)\right]$ is a complete graph $K^{v}$ on at least three vertices. Let $B^{v}$ be an $M$-basis for $K^{v}$. By Lemma 3.1, we may extend $B^{v}$ to an $M$-basis $B$ for $C$, in which each vertex $w \in W$ is incident to exactly three edges of $B$ and there are no edges of $B$ between vertices in $W$. Since $C$ is rigid, Lemma 3.1 now implies that $C-W$ is also rigid. This argument may be repeated for each group of vertices $W^{\prime} \subseteq Y-X$ with a common neighbour $v^{\prime} \in X$ to deduce that $G[X]^{2}=G^{2}[X]=C-(Y-X)$ is rigid. Theorem 2.3 now implies that $\operatorname{def}(G[X])=0$ and hence $G[X]$ is strong.

We showed in [10] that if two strong subgraphs have a non-empty intersection, then their union is strong. Together with Lemma 5.1, this implies that the partition $\mathcal{Q}$ of $V$ generated by the rigid components of $G^{2}$ is a refinement of the brick partition $\mathcal{B}$ of $G$, when $G$ has minimum degree at least two.

Lemma 5.2 Let $G$ be a graph of minimum degree at least two, $B=G[X]$ be a strong subgraph of $G$ and $Y=X \cup N_{G}(X)$. Suppose that Conjecture 2.1 holds for $B$. Then $G[Y]^{2}$ is rigid.

Proof: If $|X|=1$ then $G[Y]^{2}$ is a complete graph, and hence is rigid. Suppose $|X| \geq 3$. Since $B$ is strong, $B$ has minimum degree at least two. By Conjecture 2.1, $G[X]^{2}$ is rigid. Thus $G[Y]^{2}$ is rigid by Lemma 3.1.

Lemma 5.3 Let $G$ be a graph of minimum degree at least two.

(a) Let $B=G[X]$ be a brick of $G, Y=X \cup N_{G}(X)$, and suppose that Conjecture 2.1 holds for B. Then $G[Y]^{2}$ is a rigid component of $G^{2}$. 
(b) Let $C$ be a rigid component of $G^{2}, Y=V(C)$, and $X=Y-V_{1}(G[Y])$. Let $B$ be the brick of $G$ which contains $G[X]$ and suppose that Conjecture 2.1 holds for $B$. Then $B=G[X]$.

Proof: (a) Since $G[X]$ is a brick, each vertex in $X$ has degree at least two in $G[Y]$ (if $X$ is non-trivial, each vertex has degree at least two already in $G[X]$; if $X$ is trivial, it follows from the fact that $G$ has minimum degree at least two). By Lemma 5.2, G[Y] ${ }^{2}$ is rigid. Let $C$ be the rigid component of $G^{2}$ containing $G[Y]^{2}$ and let $Y^{\prime}=V(C)$. Suppose $Y^{\prime}-Y \neq \emptyset$. By Lemma 5.1, $X^{\prime}=Y^{\prime}-V_{1}\left(G\left[Y^{\prime}\right]\right)$ is strong. Now $X \subseteq X^{\prime}$ since $X \cap V_{1}\left(G\left[Y^{\prime}\right]\right)=\emptyset$. Since $X$ is a brick, we must have $X=X^{\prime}$ and hence $Y=Y^{\prime}$. Thus $G^{2}[Y]$ is a rigid component of $G^{2}$. Since $B$ is a brick, it is easy to see that $G^{2}[Y]=G[Y]^{2}$.

(b) Since $C$ is a rigid component of $G^{2}$, Lemma 5.1 implies that $G[X]$ is strong. Let $X^{\prime}=V(B)$. By Lemma 5.2, $G\left[X^{\prime} \cup N_{G}\left(X^{\prime}\right)\right]^{2}$ is rigid. Since $C$ is a rigid component of $G^{2}$, we have $|Y| \geq 3$ by Lemma 4.1(a). Since $Y \subseteq X^{\prime} \cup N_{G}\left(X^{\prime}\right)$ and $C$ is a rigid component, we have $X^{\prime} \cup N_{G}\left(X^{\prime}\right)=Y$. Thus $X^{\prime} \subseteq Y$. But $d_{G[Y]}(v)=1$ for all $v \in Y-X$, since $Y-X=V_{1}(G[Y])$. Since $X^{\prime} \subseteq Y$ and $G\left[X^{\prime}\right]$ is a brick, we must have $v \notin X^{\prime}$ for all $v \in Y-X$. Thus $X^{\prime}=X$ and $B=G[X]$.

Lemmas 5.2 and 5.3 immediately imply:

Theorem 5.4 Suppose that Conjecture 2.1 holds. Let $G=(V, E)$ be a graph of minimum degree at least two. Then for each rigid component $C$ of $G^{2}$ there is a brick $B=G[X]$ of $G$ with $C=G\left[X \cup N_{G}(X)\right]^{2}$, and for each brick $B=G[X]$ of $G$ the subgraph $G\left[X \cup N_{G}(X)\right]^{2}$ is a rigid component of $G^{2}$.

Theorem 5.4 immediately implies that if Conjecture 2.1 holds then the partition $\mathcal{Q}$ of $V$ generated by the rigid components of $G^{2}$ is identical to the brick partition $\mathcal{B}$ of $G$, when $G$ has minimum degree at least two. We may now deduce:

Corollary 5.5 Conjectures 2.1, 2.2 and 4.5 are all equivalent. Furthermore they would each follow from the truth of Conjectures 3.4 and 3.5.

Proof: The fact that Conjectures 2.1 and 2.2 are equivalent is a result in [10]. We show that Conjectures 2.2 and 4.5 are equivalent. By Lemma 4.6, it will suffice to show that they are equivalent for graphs of minimum degree 
at least two. Let $G=(V, E)$ be a graph of minimum degree at least two. Let $\mathcal{B}$ be the brick partition of $G, \mathcal{C}$ be the set of rigid components of $G^{2}$, and $\mathcal{Q}$ be the partition of $V$ generated by $\mathcal{C}$.

Suppose Conjecture 2.2 holds. Then $r\left(G^{2}\right)=3|V|-6-\operatorname{def}(G)$. Since $\operatorname{def}_{G}(\mathcal{B})=\operatorname{def}(G)$ by $[10], \mathcal{B}=\mathcal{Q}$ by the above, and $3|V|-6-\operatorname{def}_{G}(\mathcal{Q})=$ $\operatorname{val}(\mathcal{C})$ by Lemma $4.4(\mathrm{~b})$, we have $r\left(G^{2}\right)=\operatorname{val}(\mathcal{C})$. Thus Conjecture 4.5 holds for $G$.

Suppose, on the other hand, that Conjecture 4.5 holds. Then

$$
r\left(G^{2}\right)=\operatorname{val}(\mathcal{C})=3|V|-6-\operatorname{def}_{G}(\mathcal{Q}) \geq 3|V|-6-\operatorname{def}(G) \geq r\left(G^{2}\right),
$$

by Lemma 4.4(b), and Theorem 2.3. Thus equality holds throughout and Conjecture 2.2 holds for $G$.

It is easy to see that Conjectures 3.4 and 3.5 would imply Conjecture 4.5 .

It was shown in [10] that, if true, Conjecture 2.2 could be used to determine the rank of squares of all graphs, not just graphs of minimum degree at least two. It is also possible to extend the results of this section to squares of arbitrary graphs. We omit the details.

A graph $H$ is called redundantly rigid if $H-e$ is rigid for all $e \in E(H)$. In applications it is sometimes useful to identify the redundantly rigid components (that is, the maximal redundantly rigid subgraphs) of a molecular graph, see [12]. It would be interesting to find (possibly assuming the truth of Conjecture 2.1) a connection between the redundantly rigid components of $G^{2}$ and the maximal 'superstrong' subgraphs of $G$, where a subgraph $F$ of $G$ is superstrong if $5 F-e$ has six edge-disjoint spanning trees for all $e \in E(5 F)$. As a first step, one may ask when the square of a superstrong graph is redundantly rigid. This is not always the case. Consider, for example, the graph $G_{0}$ consisting of two 4 -cycles joined at a cut-vertex. Then $G_{0}$ is superstrong but $G_{0}^{2}$ is not redundantly rigid since it contains vertices of degree three. It is conceivable, however, that if $G$ is superstrong and $G^{2}$ has minimum degree at least four then $G^{2}$ is redundantly rigid.

\section{The algorithm}

Conjecture 2.1 and Theorem 5.4 have important algorithmic consequences. The deficiency and the brick partition of a graph $G$ can be found in polynomial time (see below). Thus, provided Conjecture 2.1 is true, we can 
compute the number of degrees of freedom and the rigid components of $G^{2}$ by a fast combinatorial algorithm. This algorithm will run on the graph $5 G .^{5}$

The fact that the brick partition of a graph can be computed in polynomial time can be seen from the following more general argument. Given a multigraph $H=(V, E)$ we define $H$ to be $k$-strong, for a positive integer $k$, if $H$ has $k$ edge-disjoint spanning trees. The $k$-brick partition of $H$ is the partition of $V$ into the vertex sets of the maximal $k$-strong subgraphs of $H$. We may find the $k$-brick partition of $H$ as follows.

STEP 1 Construct a maximum size spanning subgraph $H^{\prime}=(V, I)$ of $H$ whose edge set can be partitioned into $k$ forests.

STEP 2 For each $v \in V$ construct the $k$-brick of $H^{\prime}$ which contains $v$. The subgraphs of $H$ induced by the vertex sets of these $k$-bricks are the $k$-bricks of $H$.

Step 1 is the same as finding a basis $I$ in the matroid $\mathcal{M}_{k}(H)$, which is the matroid union of $k$ copies of the cycle matroid of $H$. An efficient algorithm for this is given by Gabow and Westermann in [4]. Since $I$ is independent in $\mathcal{M}_{k}(H)$, we have $\left|E_{H^{\prime}}(X)\right| \leq k|X|-k$ for all nonempty $X \subseteq V$. Thus finding a $k$-brick of $H^{\prime}$ containing a specified edge $v w \in I$ in Step 2 is equivalent to finding a maximal subset $B \subseteq V$ which contains both $v$ and $w$ and satisfies $\left|E_{H^{\prime}}(B)\right|=k|B|-k$. This subroutine is easy to implement by maximum flow (or bipartite matching, or degree constrained orientation) algorithms. If no such subgraph exists we choose another edge incident to $v$ and repeat the subroutine until we either find the $k$-brick of $H^{\prime}$ containing $v$, or all edges of $I$ incident to $v$ have been exhausted. In the latter case we can conclude that the $k$-brick of $H^{\prime}$ containing $v$ is just the isolated vertex $v$. The assertion that the vertex sets of the $k$-bricks of $H^{\prime}$ and $H$ are the same follows from the fact that, for each circuit $C$ of $\mathcal{M}_{k}(H)$, and each $e \in C$, the subgraph of $H$ induced by $C-e$ is $k$-strong (c.f. [20, Proposition A.1.1]).

There exist more efficient methods for finding the $k$-brick partition of

\footnotetext{
${ }^{5}$ The algorithm of Jacobs $[11,12]$, which runs on $G^{2}$, computes the number of degrees of freedom by building up a maximal (with respect to inclusion) subset $F$ of edges of $G^{2}$ in a greedy fashion with the property that no subset $X \subseteq V$ with $|X| \geq 3$ induces more than $3|X|-6$ edges of $F$. (This property can be tested efficiently by a version of the 'pebble game'.) It is conjectured that $F$ is $M$-independent, and that $F$ is a maximum size $M$-independent edge set in $G^{2}$. Even though computational results are convincing, there is no rigorous proof for its correctness, even if we assume that Conjecture 2.1 is true. With some additional computations the algorithm identifies the maximal rigid subgraphs of $G^{2}$ in a similar manner.
} 
$H$, where the basis $I$ and the $k$-brick partition are built up simultaneously. We omit the details and refer the reader to [15, Chapter 51] for a detailed survey and $[1,24]$ for the orientation-based approach and more references.

We may obtain the brick partition for a graph $G$ by applying the above algorithm to find the 6 -brick partition for the graph $H=5 G$. The set $I$ constructed in Step 1 satisfies $|I|=6|V|-6-\operatorname{def}(G)$. Thus, if Conjecture 2.2 (or equivalently Conjecture 2.1 ) were true, and $G$ has minimum degree at least two, then the number of degrees of freedom of $G^{2}$ would be $6|V|-|I|$. Furthermore Theorem 5.4 implies that the rigid components of $G^{2}$ would be the subgraphs $G^{2}\left[X \cup N_{G}(X)\right]$ with $X=V(B)$ for each brick $B$ of $G$.

It is straightforward to adapt the above procedure to handle the case when $G$ has vertices of degree one.

\section{References}

[1] A.R. Berg and T. Jordán, Algorithms for graph rigidity and scene analysis, Algorithms - ESA 2003, Lecture Notes in Comput. Sci., 2832, Springer, Berlin, 78-89, 2003.

[2] H. Crapo, A. Dress and T.-S. Tay, Problem 4.2, in Matroid Theory (J.E. Bonin, J.G. Oxley and B. Servatius eds., Seattle, WA, 1995), Contemp. Math., 197, Amer. Math. Soc., Providence, RI, 1996, 414.

[3] D. Franzblau, Generic rigidity of molecular graphs via ear decomposition, Discrete Applied Math. 101 (2000) 131-155.

[4] H.N. Gabow and H.H. Westermann, Forests, frames, and games: algorithms for matroid sums and applications, Algorithmica, Vol. 7, pp. 465-497, 1992.

[5] H. GLuck, Almost all simply connected surfaces are rigid, Geometric topology, Lecture Notes in Mathematics No. 438, Springer, 1975, pp. 225-239.

[6] J. Graver, B. Servatius, and H. Servatius, Combinatorial Rigidity, AMS Graduate Studies in Mathematics Vol. 2, 1993.

[7] B. JaCkson And T. Jordán, The $d$-dimensional rigidity matroid of sparse graphs, J. Combin. Theory Ser. B 95 (2005), no. 1, 118-133. 
[8] B. Jackson and T. Jordán, The Dress conjectures on rank in the 3-dimensional rigidity matroid, Adv. in Appl. Math. 35 (2005), no. 4, $355-367$.

[9] B. JaCKSON And T. Jordán, On the rank function of the 3dimensional rigidity matroid, Int. J. Comput. Geom. Appl., to appear. (see also: EGRES TR-2005-09, www.cs.elte.hu/egres/)

[10] B. JaCKSON And T. JordÁn, Rank and independence in the rigidity matroid of molecular graphs, submitted. (see also: EGRES TR-2006-02, www.cs.elte.hu/egres/)

[11] D. JaCoBs, Generic rigidity in three-dimensional bond-bending networks, J. Phys. A, 31, 6653-6668 (1998).

[12] D. Jacobs, L.A. Kunn, And M.F. Thorpe, Flexible and rigid regions in proteins, in: Rigidity theory and applications (Edited by Thorpe and Duxbury), Kluwer 1999, pp. 357-384.

[13] D.J. Jacobs, A.J. Rader, L.A. Kuhn, And M.F. Thorpe, Protein flexibility predictions using graph theory, PROTEINS: Structure, Function, and Genetics, 44:150-165 (2001).

[14] C. St. J. A. Nash-Williams, Edge-disjoint spanning trees of finite graphs, J. London Math. Soc. 36 (1961) 445-450.

[15] A. Schrijver, Combinatorial Optimization, Springer, 2003.

[16] T-S. TAY, On the generic rigidity of bar frameworks, Advances in Applied Mathematics, 23, (1999), 14-28.

[17] T.S. TAY and W. Whiteley, Recent advances in the generic rigidity of structures, Structural Topology 9, 1984, pp. 31-38.

[18] M.F. Thorpe, M. Chubynsky, B. Hespenheide, S. Menor, D.J. Jacobs, L.A. Kuhn, M.I. Zavodszky, M. Lei, A.J. Rader, W. Whiteley, Flexibility in biomolecules, in: Current topics in physics (R.A. Barrio and K.K. Kaski, eds.), Imperial College Press, London, 2005, Chapter 6, 97-112.

[19] W.T. TutTe, On the problem of decomposing a graph into $n$ connected factors, J. London Math. Soc. 36 (1961) 221-230. 
[20] W. Whiteley, Some matroids from discrete applied geometry, in Matroid theory (J.E. Bonin, J.G. Oxley and B. Servatius eds., Seattle, WA, 1995), Contemp. Math., 197, Amer. Math. Soc., Providence, RI, 1996, 171-311.

[21] W. Whiteley, Rigidity of molecular structures: geometric and generic analysis, in: Rigidity theory and applications (Edited by Thorpe and Duxbury), Kluwer 1999, pp. 21-46.

[22] W. Whiteley, Rigidity and scene analysis, in: Handbook of Discrete and Computational Geometry (J. E. Goodman and J. O'Rourke, eds.), CRC Press, Second Edition, pp. 1327-1354, 2004.

[23] W. Whiteley, The equivalence of molecular rigidity models as geometric and generic graphs, manuscript, 2004.

[24] W. Whiteley, Counting out to the flexibility of molecules, Physical Biology 2 (2005) S116-S126. 